\title{
Resident Perspectives on COVID-19 in Psychiatric Decision-Making
}

\author{
Amy Gallop $^{1}$ (D) - Matthew R. Graczyk ${ }^{1}$
}

Received: 29 May 2020 / Accepted: 8 September 2020/Published online: 23 September 2020

(C) Academic Psychiatry 2020

To the Editor:

As we left our shifts on the evening of Mr. L's discharge, we began to worry. We wondered about the likelihood of him maintaining his sobriety and finding stable housing. How likely was it that he would be able to access and navigate the technology required to connect with mental health and substance use disorder treatment? Would the shelter he was discharged to eventually close due to viral spread? The challenges of ensuring safe disposition for patients have increased with COVID-19. Mr. L's situation is not unique.

As two first year psychiatry residents at a busy urban hospital, we have seen how the pathogenesis of COVID-19 has disproportionately targeted our patients with psychiatric illness. We have found ourselves at a crossroads, wondering how we can provide safe and thorough care in the age of social distancing.

Mr. L was an elderly gentleman with a history of depression, who presented to our emergency department with acute suicidality in the setting of cocaine withdrawal. He was homeless and had been residing in various shelters and group homes with known positive coronavirus cases. Although he was clinically asymptomatic, his chronic substance use rendered him unable to confidently deny COVID-19 symptoms over the prior week.

Triaging Mr. L in the pre-COVID era would have been rather unremarkable. He would have been admitted to inpatient psychiatry, and after time away from substance use, he could have reinitiated pharmacotherapy, participated in individual/group therapy, and ultimately would have been discharged armed with support to help him find housing and address his substance use.

Rapidly changing testing guidelines, limited testing resources, and slow turnaround time for COVID-19 labs have

Amy Gallop

amy.gallop@health.slu.edu

1 St. Louis University Hospital, St. Louis, MO, USA resulted in long waits in the emergency department. Mr. L spent over twenty-four hours in the ED awaiting medical clearance prior to admission to inpatient psychiatry. Although we could help him comfortably get through the withdrawal period and initiate psychotropic medications, the environment was not therapeutic. He did not have daily social work meetings, family meetings, group therapy, or therapeutic interactions with other patients during this period.

Oftentimes, patients with mental illness present to the emergency department during some of the lowest points of their lives, feeling isolated, hopeless, and worthless. Many of them have multiple risk factors for COVID, and screening results in an extended emergency department stay while awaiting test results. They spend sleepless hours listening to the buzz of the emergency department, where day and night are indistinguishable, and they wait in a small room alone without distraction from their thoughts.

After a negative COVID-19 test, Mr. L was admitted to the psychiatric unit. Through participation in the therapeutic milieu (group therapy, individual therapy, daily meetings with social work, and our psychiatrists), his psychiatric symptoms improved. He attributed this improvement to the antidepressant that was initiated days earlier, but it was evident that the social support he received while admitted along with maintenance of his sobriety played a crucial role in his improvement.

For patients dealing with substance use, after a brief stay on a psychiatric unit, inpatient or intensive outpatient substance use disorder treatment will provide the highest chance of success. There are now policies on inpatient treatment to reduce COVID-19 spread, resulting in decreased access. Many treatment centers are now offering telemedicine services for substance use. Our homeless population struggling with substance use disorders often have limited access to technology that would facilitate this treatment.

Prior to COVID-19, Mr. L would likely have met with his caseworker before discharge, who would have continued to meet with him weekly thereafter on discharge. He would have been transferred to an inpatient substance use disorder treatment setting, where he would have gained tools and support to 
maintain his sobriety. While there, he would have continued to meet with social workers to identify housing options.

Instead, Mr. L was discharged to a homeless shelter and referred to substance use disorder treatment, where he would have a virtual intake meeting. He was to follow up at a federally qualified health center for a virtual psychiatry meeting. We remain guarded about Mr. L's long-term prognosis.

Within the first weeks of our rotations on the psychiatric unit, we realized the importance of becoming familiar with the region's resources for people struggling with homelessness, lack of financial stability, addiction, and serious mental illness. These resources are crucial to facilitate a patient's safe disposition. With the COVID-19 pandemic, access is more challenging.

As residents tasked with learning to account for both the psychosocial considerations when working with a patient, we find this to be disheartening. We remain hopeful, though. We have increased our telemedicine service and believe this presence will continue into the future. This will allow increased access for patients with travel barriers. We have also initiated pre-appointment calls to educate patients on the new technology. As residents, we have adapted a new style of communication that is compatible with life in front of a camera. We now rely more heavily on patient history and collateral sources rather than non-verbal cues during patient encounters.

Going forward, interdisciplinary collaboration is most important. We must be aware of the unique disposition challenges faced by patients with mental illness and work to understand the resources available in our area. Due to the increased use of telemedicine services since COVID-19 began, we now have systems in place that make interdisciplinary communication more accessible and will continue to play a role in psychiatric care in the future. Team meetings between a patient, his caseworker, substance use counselor, and psychiatrists can occur more regularly and efficiently. Multiple churches in our region are installing telemedicine hubs for people without access to computers to continue to receive psychiatric care and continued efforts to improve access to technology will be crucial in increasing availability of care. Although social distancing during COVID-19 has caused many people to feel disconnected and isolated, we are hopeful that this utilization of telemedicine will increase access to mental health services and reach people who have not had access in the past.

\section{Compliance with Ethical Standards}

Conflict of Interest The authors declare that they have no conflicts of interest.

Disclosures Names and identifying details have been changed to protect the privacy of individuals.

This piece is based on our own views and does not represent the institution for which we are employed as resident physicians.

Publisher's Note Springer Nature remains neutral with regard to jurisdictional claims in published maps and institutional affiliations. 